\title{
Anti-inflammatory Activity of Standardized Fraction from Inula helenium L. via Suppression of NF-KB Pathway in RAW 264.7 Cells
}

\author{
Jaemoo Chun ${ }^{1,2}$, Kwangho Song ${ }^{1}$, and Yeong Shik Kim ${ }^{1, *}$ \\ ${ }^{l}$ Natural Products Research Institute, College of Pharmacy, Seoul National University, Seoul 08826, Republic of Korea \\ ${ }^{2}$ Current Address: Winship Cancer Institute, Emory University School of Medicine, Atlanta, GA 30322, USA
}

\begin{abstract}
Inula helenium L. is rich source of eudesmane-type sesquiterpene lactones, mainly alantolactone and isoalantolactone, which have the various pharmacological functions. In this study, we examined the inhibitory effects of nitric oxide (NO) production of hexane, methylene chloride, ethyl acetate, butanol, and water fractions from I. helenium and investigated the anti-inflammatory effect of hexane fraction of I. helenium (HFIH) in LPSinduced RAW 264.7 cells. Quantification of alantolactone and isoalantolactone from HFIH was carried out for the standardization by multiple reaction monitoring using triple quadrupole mass spectrometer. HFIH significantly inhibited inducible NO synthase (iNOS) and cyclooxygenase-2 (COX-2) protein as well as their downstream products $\mathrm{NO}$ and prostaglandin $\mathrm{E}_{2}\left(\mathrm{PGE}_{2}\right)$ in LPS-stimulated RAW 264.7 cells. Moreover, HFIH suppressed NF$\kappa \mathrm{B}$ transcriptional activity by decreasing the translocation of p65 to the nucleus. The in vivo study further confirmed that HFIH attenuated the paw edema induced by carrageenan in an acute inflammation model. These findings suggest that HFIH may be useful as a promising phytomedicine for inflammatory-associated diseases. Keywords - Inula helenium L., Sesquiterpene lactones, Anti-inflammation, NF-אB, RAW 264.7 cells
\end{abstract}

\section{Introduction}

Inflammation is a complex biological process which is regulated by various inflammatory mediators and cytokines produced by activated macrophages. ${ }^{1}$ Many inflammatory diseases such as rheumatoid arthritis, asthma, and inflammatory bowel diseases occur when the regulation of inflammatory process was disrupted. Macrophages are the main pro-inflammatory cells which respond to invading pathogens by releasing inflammatory mediators including nitric oxide (NO) produced by inducible NO synthase (iNOS) and prostaglandin $\mathrm{E}_{2}\left(\mathrm{PGE}_{2}\right)$ produced by cyclooxygenase-2 (COX-2). High levels of NO and $\mathrm{PGE}_{2}$ were found in these inflammatory diseases. Therefore, suppressing the production of inflammatory mediators such as $\mathrm{NO}$ and $\mathrm{PGE}_{2}$ could be preventive or therapeutic strategy for inflammatory diseases. ${ }^{2}$

Nuclear factor $\kappa \mathrm{B}(\mathrm{NF}-\kappa \mathrm{B})$ plays a crucial role in the regulation of mediators involved in immune and inflammatory responses. ${ }^{3}$ In unstimulated, NF- $\mathrm{kB}$ interacts with the inhibitory $\kappa \mathrm{B} \alpha(\mathrm{I} \kappa \mathrm{B} \alpha)$ protein in the cytosol. NF- $\kappa \mathrm{B}$

\footnotetext{
*Author for correspondence

Dr. Yeong Shik Kim, Natural Products Research Institute, College of Pharmacy, Seoul National University, 1 Gwanak-ro, Gwanak-gu, Seoul 08826, Republic of Korea.

Tel: +82-2-880-2479; E-mail: kims@snu.ac.kr
}

is activated by a range of stimuli, which trigger

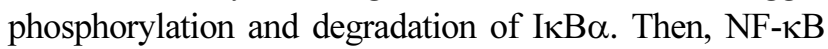
$\mathrm{p} 65 / \mathrm{p} 50$ heterodimer translocate from the cytosol to the nucleus, where it binds to its promoter and activates transcription of pro-inflammatory target genes including iNOS and COX-2. Thus, down-regulation of NF-kB activation would be a potential therapeutic strategy to treat or prevent the NF-кB-related human inflammatory diseases. $^{4}$

There has been an increase in the use of medicinal plants worldwide to develop the anti-inflammatory medicines derived from natural products, which are far less likely to cause adverse side effects, unlike chemically synthesized substances. ${ }^{5}$ Inula helenium L. (Elecampane) is a medicinal plant widely distributed throughout Europe and East Asia. ${ }^{6}$ The roots of $I$. helenium have been widely used as anti-inflammatory, anti-bacterial, and anti-tumor agents. It has been demonstrated that the extracts of I. helenium are rich source of eudesmane-type sesquiterpene lactones. ${ }^{7}$ Several studies have shown that one of the common roles of sesquiterpene lactones is to suppress NF- $\mathrm{BB}$ pathway. ${ }^{8}$ Specifically, alantolactone and isoalantolactone have been known to suppress iNOS and COX2 expressions by inhibiting NF-kB pathway. ${ }^{9,10}$ Although a large number of sesquiterpene lactones are found in a member of the 
Asteraceae (Compositae) family, alantolactone and isoalantolatone are only found in rare plants and exist in extreme low concentrations. There has been reported that I. helenium and Inula racemosa Hook. f. are plentiful source of these compounds. ${ }^{11}$ Recently, sesquiterpene lactone-containing extracts of I. helenium has potentials in rheumatoid arthritis. ${ }^{12}$ Therefore, the anti-inflammatory effect of I. helenium may have the potential to be developed as a preventive or therapeutic drug. In addition, the development of standardized method and biological standardization of phytomedicine can provide useful information for the development of phytomedicine. In this study, we prepared methanol extract of I. helenium (MEIH) and its hexane, methylene chloride, ethyl acetate, butanol, and water fractions and investigated the antiinflammatory effects in LPS-induced RAW 264.7 cells.

\section{Experimental}

Chemicals and reagents -3-[4,5-dimethylthiazol-2yl]-2,5-diphenyltetrazolium bromide (MTT), LPS from
Escherichia coli, Griess reagent, 2-amino-5,6-dihydro-6methyl-4H-1,3-thiazine (AMT), Celecoxib, and $N$-tosyl-Lphenylalanyl chloromethyl ketone (TPCK) were purchased from Sigma-Aldrich (St. Louis, MO, USA). Dulbecco's modified Eagle's medium (DMEM), penicillin-streptomycin, and fetal bovine serum (FBS) were purchased from GenDepot (Barker, TX, USA). Alantolactone and isoalantolactone were purchased from Tauto Biotech (Shanghai, China). Antibodies against iNOS, COX-2, p65, PARP1, and $\beta$-actin were purchased from Santa Cruz Biotechnology (Santa Cruz, CA, USA).

Plant material and preparation of standardized fractions - The root of I. helenium was obtained from Gwangmyungdang Medicinal Herbs (Ulsan, Korea). A voucher specimen (NPRI-14-226) was deposited at the Natural Products Research Institute at Seoul National University. The root of $I$. helenium was extracted with methanol for $12 \mathrm{~h}$ and sonicated for $2 \mathrm{~h}$ at room temperature. The methanol extract was then filtered and evaporated under reduced pressure. The obtained MEIH was suspended in distilled water and partitioned sequentially
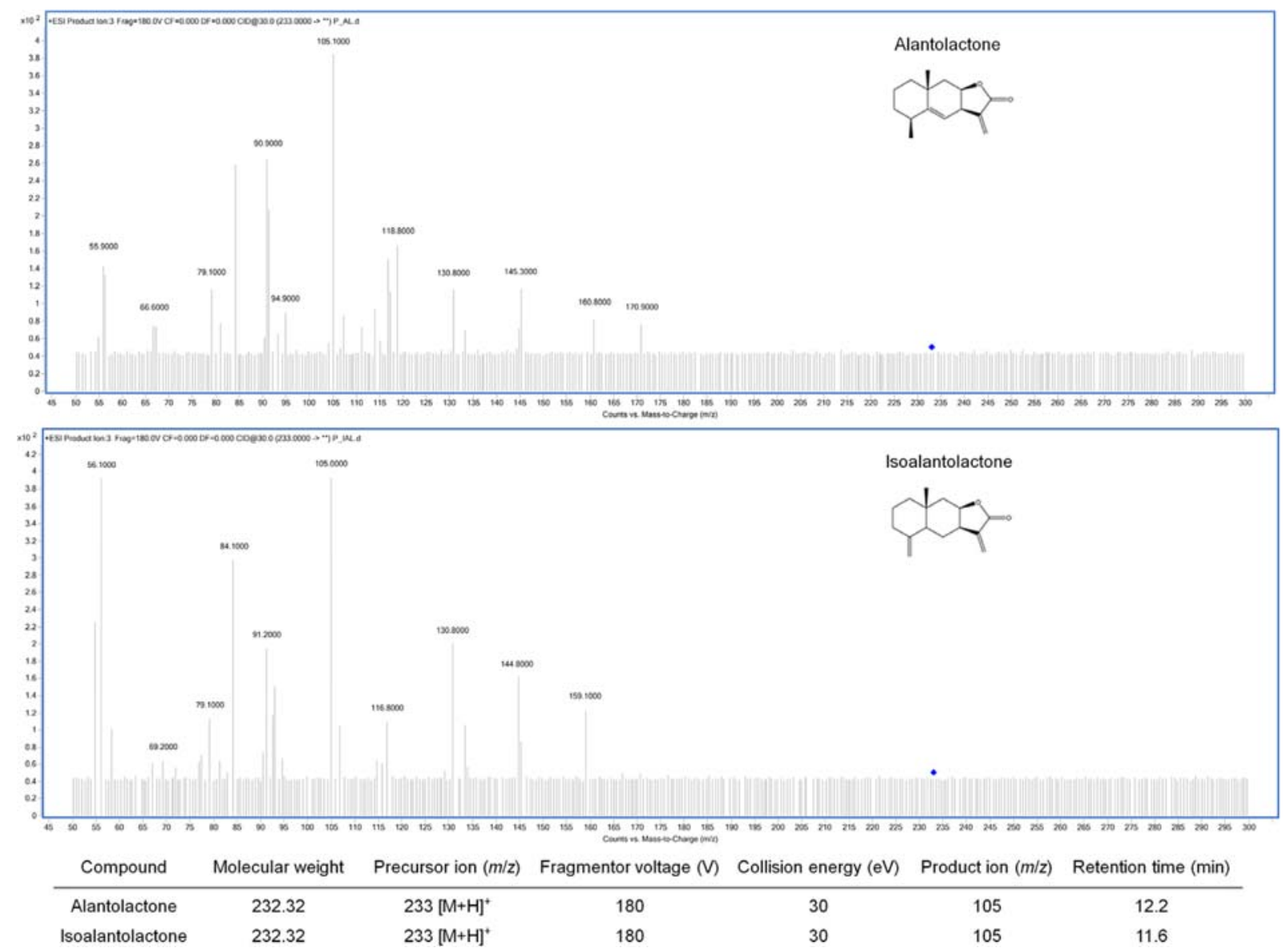

Fig. 1. Chemical structures of alantolactone and isoalantolactone and product ion scan for multiple reaction monitoring (MRM). The selected $\mathrm{m} / \mathrm{z}$ transition for alantolactone and isoalantolactone was $233 \rightarrow 105$. MRM parameters were listed. 
with hexane, methylene chloride, ethyl acetate, and butanol to obtain each fraction.

HPLC-MS/MS analysis - Quantification analysis was performed using a HPLC system coupled with triple quadrupole $(\mathrm{QqQ})$ mass spectrometer. The chromatographic separation was performed by Agilent 1260 series HPLC system (Agilent technologies, Santa Clara, CA, USA) and INNO C1 8 column $(2.0 \times 50 \mathrm{~mm}, 3 \mu \mathrm{m}$, Youngjin Biochrom, Korea) to analyze the MEIH and hexane fraction of $I$. helenium (HFIH). The mobile phase was composed of water with $0.1 \%$ formic acid as eluent $\mathrm{A}$ and acetonitrile with $0.1 \%$ formic acid as eluent $\mathrm{B}$, and the elution gradient was as follows: $0-25 \min (35-60 \% \mathrm{~B}) ; 25-30$ $\min (60-100 \% \mathrm{~B})$ at a flow rate of $0.3 \mathrm{~mL} / \mathrm{min}$. To quantify the amount of alantolactone and isoalantolactone in MEIH and HFIH, multiple reaction monitoring (MRM) analysis was performed by Agilent 6400 series triple quadrupole MS system (Agilent technologies, Santa Clara, CA, USA) and the conditions were as follows: electrospray ionization in the positive mode for the range of $\mathrm{m} / z 50-300 ; 10 \mathrm{~L} / \mathrm{min}$ and $350{ }^{\circ} \mathrm{C}$ of drying gas; 30 psi of nebulizer pressure; $4000 \mathrm{~V}$ of capillary voltage. MRM analysis is more sensitive and accurate than the HPLC detection. ${ }^{13}$ The sensitivity of the MRM mode of HPLC-QqQ-MS/MS is highly dependent upon the fragmentor voltage and collision energy. The optimal fragmentor voltage and collision energy for alantolactone and isoalantolactone were $180 \mathrm{~V}$ and $30 \mathrm{eV}$, respectively (Fig. 1). Both alantolactone and isoalantolactone provided the product ion at $m / z 105$, which was selected for MRM. The standard curves of alantolactone and isoalantolactone were obtained and had good linear correlations $\left(\mathrm{R}^{2}>0.99\right)$ (Fig. 2A). The content of alantolactone and isoalantolactone in MEIH was 44.1 and $35.1 \mathrm{mg} / \mathrm{g}$, respectively. The content of alantolactone and isoalantolactone in $\mathrm{HFIH}$ was 281.9 and $134.5 \mathrm{mg} / \mathrm{g}$, respectively (Fig. 2B).

Cell culture-RAW 264.7 macrophage cells were obtained from the American Type Culture Collection (Manassas, VA, USA). Cells were maintained in DMEM supplemented with $10 \% \mathrm{FBS}, 1 \%$ penicillin-streptomycin in a humidified atmosphere of $5 \% \mathrm{CO}_{2}$ at $37^{\circ} \mathrm{C}$. RAW 264.7 cells harboring a pNF- $\kappa \mathrm{B}$ secretory alkaline phosphatase (SEAP)-NPT reporter construct were cultured under the same conditions, except that DMEM was supplemented with $500 \mu \mathrm{g} / \mathrm{mL}$ geneticin.

Cell viability - Cell viability was assessed by MTT assay. RAW 264.7 cells were seeded into a 96-well plate at a density of $1 \times 10^{4}$ cells/well, incubated for $24 \mathrm{~h}$, and treated with various concentrations of samples for $24 \mathrm{~h}$. The MTT solution was added to each well and the
A

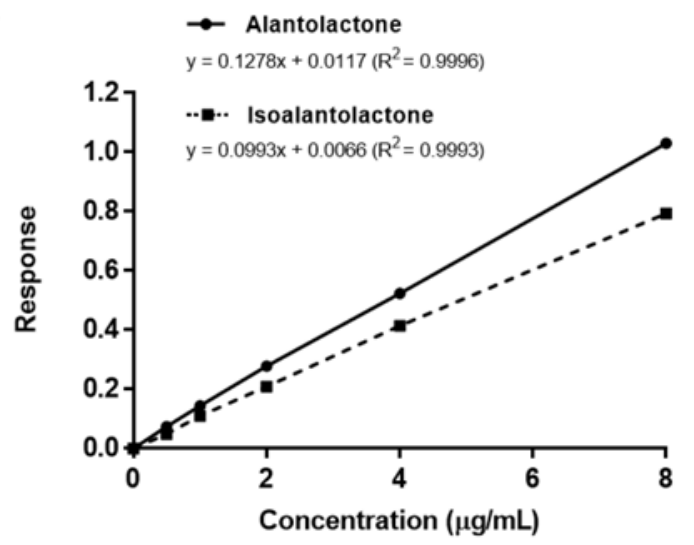

B

\begin{tabular}{ccc}
\multirow{2}{*}{ Compound } & \multicolumn{2}{c}{ Amount $(\mathrm{mg} / \mathrm{g})$} \\
\cline { 2 - 3 } & $\mathrm{MEIH}$ & $\mathrm{HFIH}$ \\
\hline Alantolactone & $44.09 \pm 0.07$ & $281.94 \pm 5.73$ \\
Isoalantolactone & $35.09 \pm 0.37$ & $134.48 \pm 4.59$
\end{tabular}

Fig. 2. Quantification of alantolactone and isoalantolactone by MRM mode of triple quadrupole mass spectrometry. (A) Calibration curves of alantolactone and isoalantolactone by MRM analysis. (B) Calculated amount of alantolactone and isoalantolactone in MEIH and HFIH.

absorbance was measured at $595 \mathrm{~nm}$ using a microplate spectrophotometer (Molecular Devices, Sunnyvale, CA, USA). The result was expressed as a percentage, relative to the untreated control.

The measurement of $\mathrm{NO}$ and $\mathrm{PGE}_{2}$ production Nitrite concentration in the culture medium was measured by a Griess reaction. RAW 264.7 cells were seeded into a 24-well plate at a density of $1 \times 10^{5}$ cells/well and incubated for $24 \mathrm{~h}$. Cells were pretreated with various concentrations of samples for $2 \mathrm{~h}$ and stimulated with LPS $(1 \mu \mathrm{g} / \mathrm{mL})$ for $18 \mathrm{~h}$. Culture medium was mixed with an equal volume of the Griess reagent. The absorbance was measured at $540 \mathrm{~nm}$ using a microplate spectrophotometer. The amount of $\mathrm{PGE}_{2}$ in the culture medium was measured using an enzyme immunoassay (EIA) kit for $\mathrm{PGE}_{2}$ (Cayman Chemical, Ann Arbor, MI, USA) according to the manufacturer's instructions. The absorbance was measured at $405 \mathrm{~nm}$ using a microplate spectrophotometer. The concentrations of $\mathrm{NO}$ and $\mathrm{PGE}_{2}$ were calculated from the standard curve. AMT $(10 \mu \mathrm{M})$ and Celecoxib $(20 \mu \mathrm{M})$ were used as positive controls.

NF- $\kappa B$ SEAP reporter gene assay - NF- $\kappa B$ transcriptional activity was measured by secretory alkaline phosphatase (SEAP) reporter gene assay as previously described. ${ }^{14}$ RAW 264.7 cells transfected with pNF-kB- 
SEAP-NPT encoding four copies of NF- $\kappa B$ binding $\kappa B$ sequence and SEAP as a reporter were seeded into 24well plates at a density of $1 \times 10^{5}$ cells/well and incubated for $24 \mathrm{~h}$. Cells were pretreated with various concentrations of samples for $2 \mathrm{~h}$ and stimulated with LPS $(1 \mu \mathrm{g} / \mathrm{mL})$ for $16 \mathrm{~h}$. The fluorescence from the product of the SEAP/ MUP reaction was measured using a Spectramax Gemini XS (Molecular Devices, Sunnyvale, CA, USA). Relative fluorescence units (RFU) were measured at an excitation wavelength of $360 \mathrm{~nm}$ and an emission wavelength of $449 \mathrm{~nm}$. TPCK $(20 \mu \mathrm{M})$ was used as a positive control.

Western blotting-For whole cell lysates, proteins were extracted using lysis buffer containing $1 \%$ NP-40, $20 \mathrm{mM}$ HEPES pH 7.6, $350 \mathrm{mM} \mathrm{NaCl}, 20 \%$ glycerol, 0.5 mM EDTA, $0.1 \mathrm{mM}$ EGTA, $50 \mathrm{mM} \mathrm{NaF}, 1 \mathrm{mM}$ DTT, $1 \mathrm{mM}$ PMSF, and protease inhibitor cocktail. For nuclear cell lysates, cells were suspended in lysis buffer containing $10 \mathrm{mM}$ HEPES $\mathrm{pH}$ 7.9, $10 \mathrm{mM} \mathrm{KCl}, 0.1 \mathrm{mM}$ EDTA, 0.1 mM EGTA, $1 \mathrm{mM}$ DTT, and $1 \mathrm{mM}$ PMSF. Then, $10 \%$ NP-40 was added to the swollen cells in the lysis buffer, followed by centrifugation for $5 \mathrm{~min}$. The nuclear pellets were suspended in nuclear extraction buffer containing 20 mM HEPES pH 7.9, $400 \mathrm{mM} \mathrm{NaCl}, 1 \mathrm{mM}$ EDTA, $1 \mathrm{mM}$ EGTA, $1 \mathrm{mM}$ DTT, $1 \mathrm{mM}$ PMSF, and protease inhibitor cocktail. Protein concentrations were determined by Bradford reagent. Equal amounts of protein were separated on 10\% SDS-polyacrylamide gel and transferred to nitrocellulose membranes, which was blocked with 5\% skim milk. For immunodetection, membranes were incubated with primary antibodies overnight and incubated with secondary antibodies conjugated with HRP for $1 \mathrm{~h}$. The bands were visualized by chemiluminescence kit (Intron Biotechnology, Seoul, Korea) and analyzed by LAS-1000 image analyzer (Fujifilm, Tokyo, Japan). Protein expressions are quantified using $\beta$-actin and PARP1 as internal controls. Densitometry was performed using ImageJ analysis software (NIH, Bethesda, MD, USA).

Carrageenan-induced paw edema test in mice - Paw edema was induced by a subplantar injection of $50 \mu \mathrm{L}$ of carrageenan $(1 \%$ in saline) into the right hind paw of mice. 6 male ICR mice (Samtako Bio Korea, Osan, Korea) per group were used for the experiment. Animal care and all the procedures were conducted in accordance with the guidelines of Seoul National University Institutional Animal Care and Use Committees. $2 \mathrm{~h}$ prior to carrageenan injection, the mice received intraperitoneal injection of HFIH (10 and $40 \mathrm{mg} / \mathrm{kg}$ ) and dexamethasone $(10 \mathrm{mg} / \mathrm{kg})$. Paw thickness was measured every hour for $6 \mathrm{~h}$ with a dial thickness gauge (Mitutoyo, Kawasaki, Japan). The percent increase in paw thickness was calculated

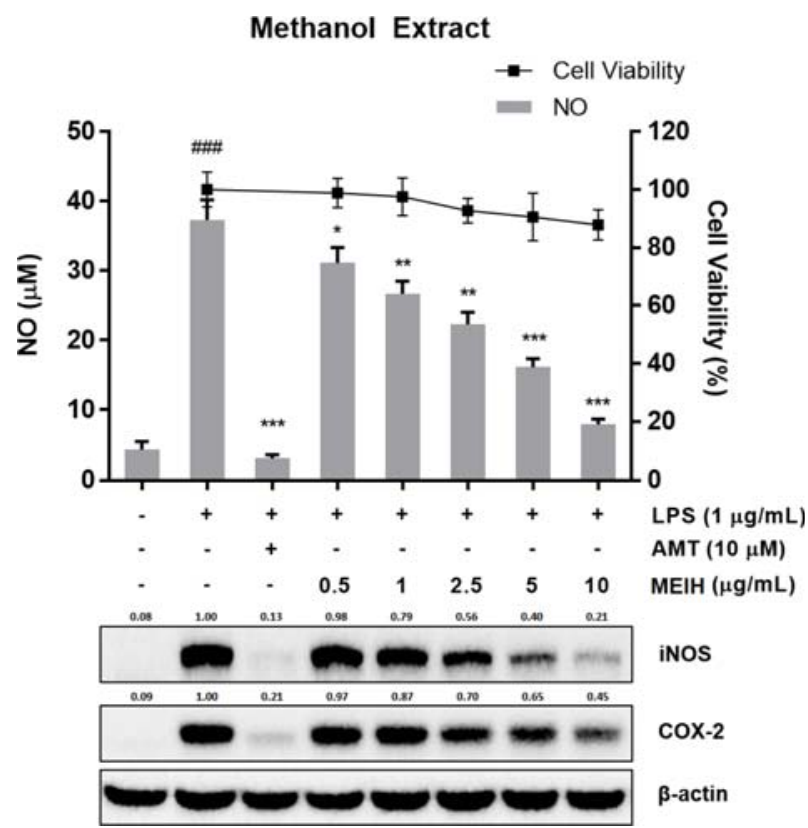

Fig. 3. Inhibitory effect of methanol extract of $I$. helenium (MEIH) on NO production and iNOS and COX-2 expression in LPS-induced RAW 264.7 cells. Cells were pretreated with MEIH for $2 \mathrm{~h}$ and stimulated for $18 \mathrm{~h}$ with LPS. Anti-proliferative effect was determined using MTT assay. The amount of NO in the medium was measured using a Griess reagent. The expression of iNOS and COX-2 was determined by Western blotting. The results are represented as the mean $\pm \mathrm{SD}(\mathrm{n}=3)$ from three independent experiments. ${ }^{\# \#} p<0.001$ indicates significant difference from the unstimulated control group. ${ }^{*} p<0.05, * * p<0.01$, and $* * * p<0.001$ indicated the significant difference compared to LPS-treated control group.

by measuring the difference between the control paw and the carrageenan-induced paw at each point.

Statistical analysis - Data were presented as means \pm $\mathrm{SD}$ of at least three independent experiments. Statistical significance $(p<0.05)$ was based on two-tailed Student's $t$ test for all the figures. Statistical analysis and graphical presentation was performed using Graphpad Prism 6.0.

\section{Result and Discussion}

To investigate whether I. helenium has anti-inflammatory effect, we examined the inhibitory effect of MEIH on the production of NO in LPS-induced RAW 264.7 cells. The used concentration of MEIH had no cytotoxicity in RAW 264.7 cells. Cells were pretreated with MEIH for $2 \mathrm{~h}$ and stimulated with LPS for $18 \mathrm{~h}$. As shown in Fig. 3, MEIH significantly inhibited LPS-induced NO production in a dose-dependent manner. Inhibition of NO production is related to the change in the protein expressions of iNOS and COX-2. ${ }^{15}$ Our results showed that LPS stimulation markedly increased the protein expressions of iNOS and 

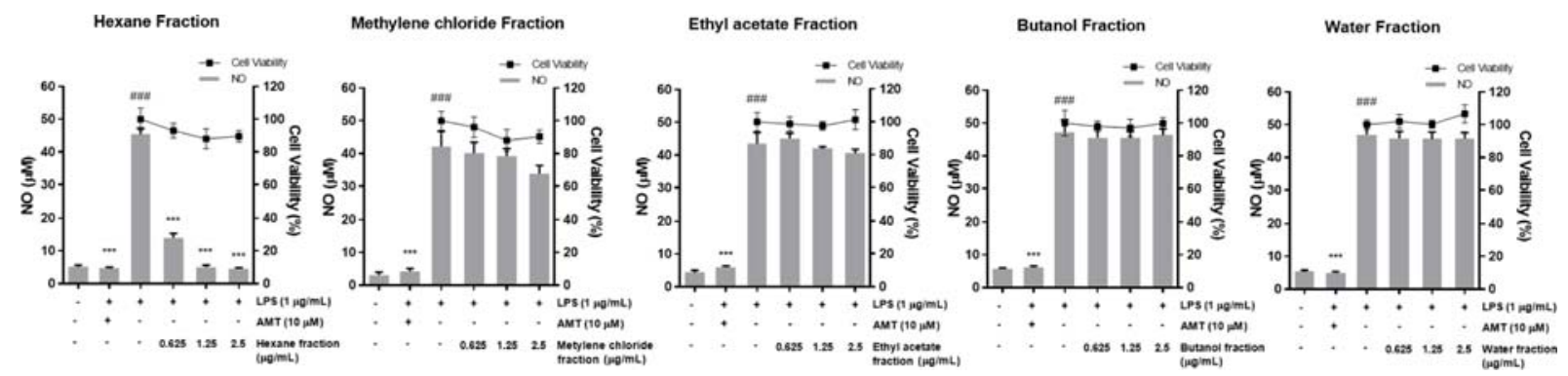

Fig. 4. Inhibitory effects of fractions from MEIH on NO production in LPS-induced RAW 264.7 cells. Cells were treated with various concentrations of fractions $(0-2.5 \mu \mathrm{g} / \mathrm{mL})$. Effects of fractions on NO production were measured by Griess reagent. The results are represented as the mean $\pm \mathrm{SD}(\mathrm{n}=3)$ from three independent experiments. \#\#\# $p<0.001$ indicates significant difference from the unstimulated control group. ${ }^{* *} p<0.001$ indicated the significant difference compared to LPS-treated control group.

COX-2. However, pretreatment with MEIH inhibited LPS-induced protein expressions of iNOS and COX-2 in a dose-dependent manner. These results suggest that MEIH exhibits anti-inflammatory properties by suppressing the protein expressions of iNOS and COX-2. Next, we used hexane, methylene chloride, ethyl acetate, butanol, and water fractions from MEIH to find the active fraction showing the anti-inflammatory effect of MEIH. Likewise, cells were pretreated with each fraction for $2 \mathrm{~h}$ and stimulated with LPS for $18 \mathrm{~h}$. As shown in Fig. 4, only HFIH significantly inhibited LPS-induced NO production in a dose-dependent manner, whereas other fractions had no effect. These data suggest that HFIH is the active fraction to exert the anti-inflammatory effect of MEIH.

We optimized the effective concentration of HFIH and further investigated whether HFIH has anti-inflammatory effect. The concentration used was determined by the effects on cell proliferation and NO production of HFIH. As shown in Fig. 5, low dose range of HFIH $(125 \mathrm{ng} / \mathrm{mL}$ to $1 \mu \mathrm{g} / \mathrm{mL}$ ) dramatically suppressed the productions of $\mathrm{NO}$ and $\mathrm{PGE}_{2}$. Likewise, pretreatment with $\mathrm{HFIH}$ inhibited LPS-induced protein expressions of iNOS and COX-2. There results suggest that HFIH could be a promising anti-inflammatory phytomedicine to treat or prevent inflammatory diseases.

$\mathrm{NF}-\mathrm{\kappa B}$ is an important pro-inflammatory mediator which controls the expression of iNOS, COX-2, and inflammatory cytokines in macrophages. ${ }^{4} \mathrm{NF}-\mathrm{\kappa B}$ transcriptional activity was measured using NF- $\mathrm{KB}$ SEAP reporter assay. RAW 264.7 cells transfected with a NF$\kappa \mathrm{B}$-mediated reporter gene construct were pretreated for 2 $\mathrm{h}$ with HFIH, followed by treatment with LPS for $16 \mathrm{~h}$. As expected, pretreatment of HFIH inhibited LPSinduced SEAP expression in a dose-dependent manner (Fig. 6A), indicating that HFIH suppresses the expression of iNOS and COX-2 through the suppression of NF-kB transcriptional activity, which controls the pro-inflammatory

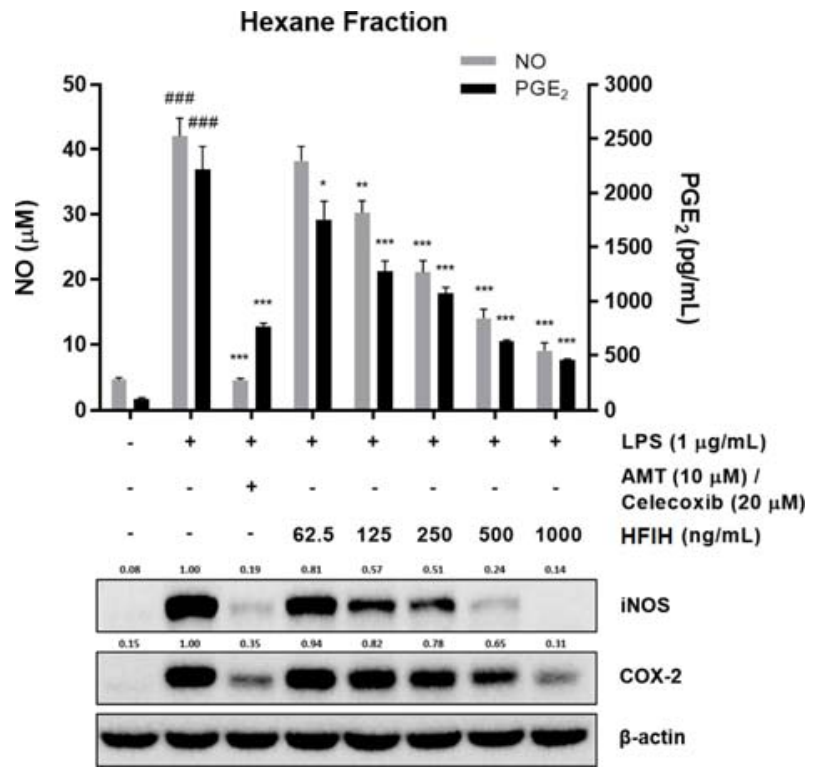

Fig. 5. Inhibitory effect of the hexane fraction of $I$. helenium $(\mathrm{HFIH})$ on the production of $\mathrm{NO}$ and $\mathrm{PGE}_{2}$ and the expression of iNOS and COX-2 in LPS-induced RAW 264.7 cells. Cells were pretreated with HFIH for $2 \mathrm{~h}$ and stimulated for $18 \mathrm{~h}$ with LPS. The amounts of $\mathrm{NO}$ and $\mathrm{PGE}_{2}$ in the medium were measured using a Griess reagent and $\mathrm{PGE}_{2}$ ELISA kit. The expression of iNOS and COX-2 was determined by Western blotting. The results are represented as the mean $\pm \mathrm{SD}(\mathrm{n}=3)$ from three independent experiments. ${ }^{\# \#} p<0.001$ indicates significant difference from the unstimulated control group. ${ }^{*} p<0.05,{ }^{*} p<0.01$, and $*_{* *} p<0.001$ indicated the significant difference compared to LPS-treated control group.

mediators in LPS-activated macrophages. Degradation of I $\mathrm{B} \alpha$ leads to NF- $\kappa \mathrm{B}$ translocation to the nucleus, where it binds to specific DNA sequences to regulate transcriptional activation of genes such as iNOS and COX-2. Thus, p65 protein localization to the nucleus is a marker of NF- $\kappa B$ activation in cells. ${ }^{16}$ As shown in Fig. $6 \mathrm{~B}$, the level of $\mathrm{p} 65$ subunit in nucleus was increased after LPS treatment. However, pretreatment with HFIH reversed this trend in a dose-dependent manner, suggesting that the 
A

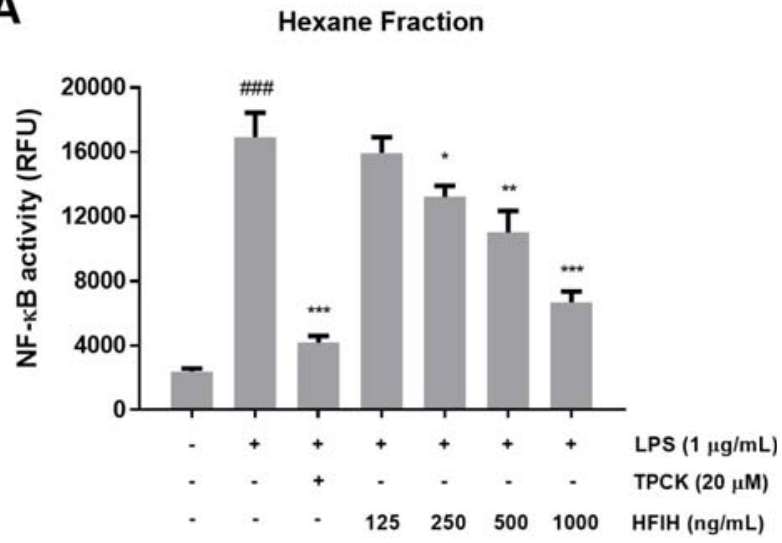

B

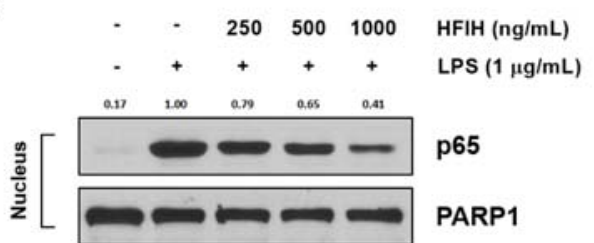

Fig. 6. Inhibitory effect of NF- $\kappa B$ activation in LPS-stimulated RAW 264.7 cells. (A) Cells harboring the NF-kB SEAP-NPT reporter construct were pretreated with the indicated concentrations of HFIH for $2 \mathrm{~h}$ then exposed to LPS. The SEAP activity was measured in RFU, using a microplate fluorometer. (B) Effect of HFIH on NF- $\kappa$ B p65 localization to the nucleus. Nuclear cell lysates were prepared for Western blotting. The results are represented as the mean $\pm \mathrm{SD}(\mathrm{n}=3)$ from three independent experiments. ${ }^{\# \#} p<0.001$ indicates significant difference from the unstimulated control group. ${ }^{*} p<0.05,{ }^{* *} p<0.01$, and $* * * p<$ 0.001 indicated the significant difference compared to LPStreated control group.

suppression of NF- $\mathrm{KB}$ transcriptional activity by HFIH was associated with the decreased translocation of $\mathrm{p} 65$ to the nucleus. PARP1 was used as a loading control for nuclear lysates.

Carrageenan-induced paw edema has been used widely as an acute animal model of inflammation. ${ }^{17}$ Therefore, we evaluated the in vivo anti-inflammatory potential of HFIH in carrageenan-induced paw edema mice model. HFIH was injected intraperitoneally $2 \mathrm{~h}$ prior to $1 \%$ carrageenan administration into the right hind paw. Dexamethasone $(10 \mathrm{mg} / \mathrm{kg})$ treatment showed the significant inhibitory effect on paw swelling for $2-6 \mathrm{~h}$. HFIH (10 and $40 \mathrm{mg} / \mathrm{kg}$ ) treatment significantly inhibited carrageenaninduced paw swelling in 2, 5, and $6 \mathrm{~h}$ points (Fig. 7). However, it is required to increase in vivo efficacy of $\mathrm{HFIH}$ in regard that alantolactone and isoalantolactone are easily metabolized in the plasma. ${ }^{18}$

Natural products are generally considered as safe and effective remedies, but their use as chemotherapeutic and chemopreventive agents is limited by several disadvantages,

\section{Carrageenan-induced paw edema}

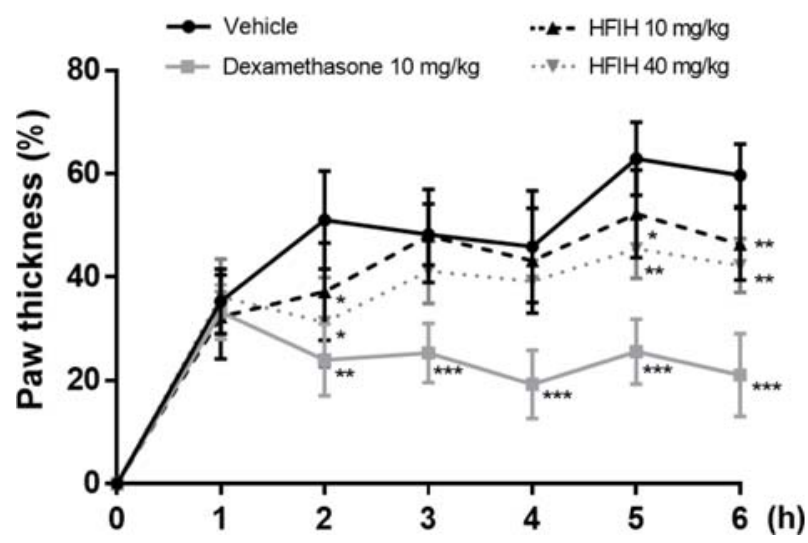

Fig. 7. Inhibitory effect of HFIH on carrageenan-induced paw edema mice model. HFIH (10 and $40 \mathrm{mg} / \mathrm{kg}$ ) was injected intraperitoneally $2 \mathrm{~h}$ prior to $1 \%$ carrageenan administration into the right hind paw. 6 mice per each group were used in this study. Paw thickness was measured every hour for $6 \mathrm{~h}$ with a dial thickness gauge. Percent increase in paw thickness was calculated by measuring the difference between the control paw and the carrageenan-induced paw at each point. Dexamethasone $(10 \mathrm{mg} /$ $\mathrm{kg}$ ) was used as a positive control. The results are represented as the mean $\pm \mathrm{SD}(\mathrm{n}=6) . * p<0.05, * * p<0.01$, and $* * * p<0.001$ indicated the significant difference compared to carrageenaninjected control group.

such as low effectiveness and difficulties of quality control. ${ }^{19}$ I. helenium is plentiful source of active sesquiterpene lactones. Specifically, hexane fraction contains alantolactone and isoalantolactone as major components. These sesquiterpene lactones have been reported to exert the anti-inflammatory effect via suppression of NF- $\mathrm{kB}$ activation in LPS-stimulated RAW 264.7 cells and peritoneal macrophages. ${ }^{9,10}$

To provide experimental data to support the antiinflammatory effect of HFIH, we investigated the inhibitory effect of alantolactone and isoalantolactone of HFIH on $\mathrm{NO}$ and $\mathrm{PGE}_{2}$ productions as well as iNOS and COX-2 expressions and compared the effects with HFIH. As shown in Fig. 8, alantolactone and isoalantolatone dosedependently inhibited LPS-induced $\mathrm{NO}$ and $\mathrm{PGE}_{2}$ productions as well as iNOS and COX2 expressions. They strongly suppressed iNOS protein expression and NO production. Although they inhibited COX-2 expression, it showed a weaker inhibitory effect than iNOS expression. Therefore, suppression of iNOS expression and $\mathrm{NO}$ production by alantolactone and isoalantolactone may play a major role in anti-inflammatory effects in RAW 264.7 cells. Further study is needed to understand the relationship between chemical structure and antiinflammatory activity of sesquiterpene lactones.

In summary, we investigated the anti-inflammatory 
A

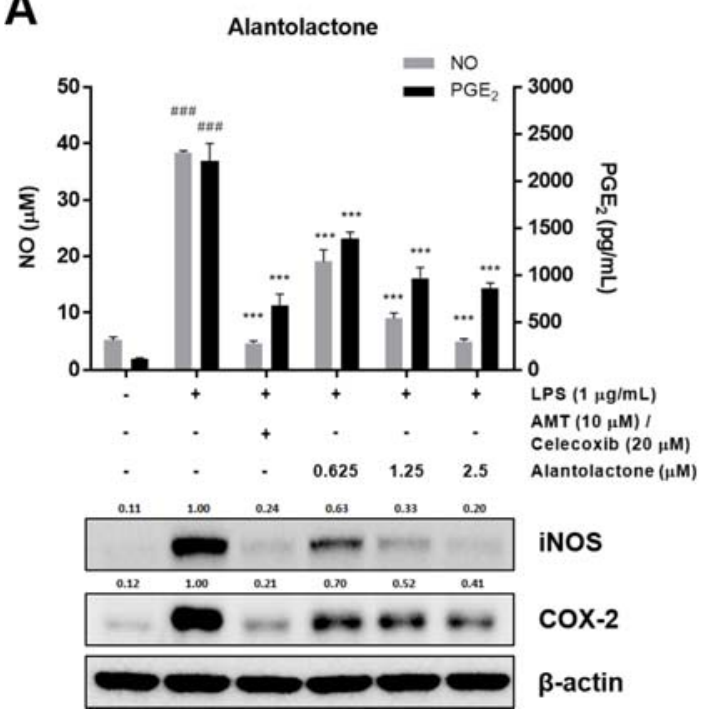

B

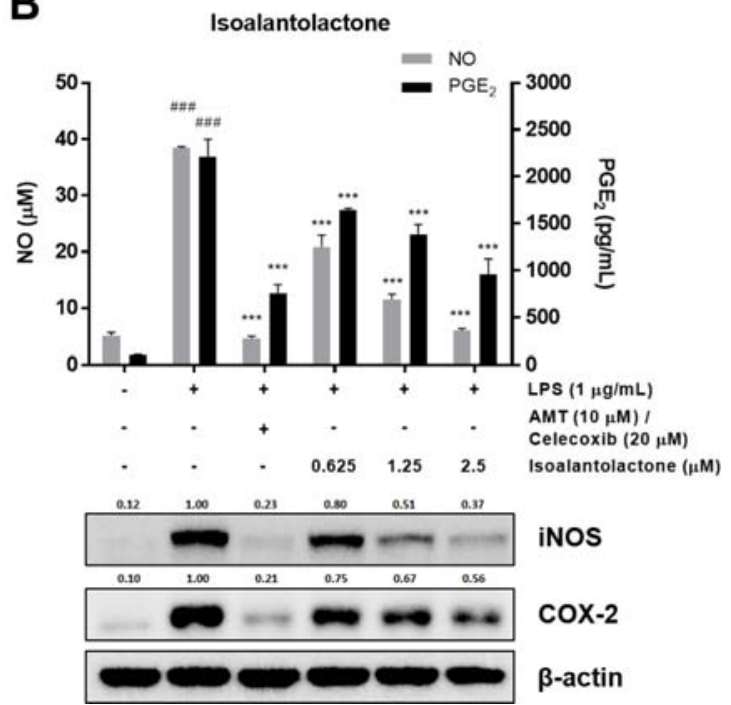

Fig. 8. Inhibitory effects of alantolactone (A) and isoalantolactone (B) on the production of $\mathrm{NO}$ and $\mathrm{PGE}_{2}$ and the expression of iNOS and COX-2 in LPS-induced RAW 264.7 cells. Cells were pretreated with compounds for $2 \mathrm{~h}$ and stimulated for $18 \mathrm{~h}$ with LPS. The amounts of $\mathrm{NO}$ and $\mathrm{PGE}_{2}$ in the medium were measured using a Griess reagent and $\mathrm{PGE}_{2}$ ELISA kit. The expression of iNOS and COX-2 was determined by Western blotting. The results are represented as the mean $\pm \mathrm{SD}(\mathrm{n}=3)$ from three independent experiments. ${ }^{\# \#} p<0.001$ indicates significant difference from the unstimulated control group. ${ }^{* * *} p<0.001$ indicated the significant difference compared to LPStreated control group.

potential of HFIH in associated with the inhibition of proinflammatory mediators $\mathrm{NO}$ and $\mathrm{PGE}_{2}$ productions in LPS-stimulated RAW 264.7 cells. We confirmed that HFIH down-regulated iNOS and COX-2 expressions via suppression of NF- $\mathrm{KB}$ activation. Moreover, HFIH inhibited carrageenan-induced paw edema in mice. Therefore, HFIH can be a potent phytomedicine for the prevention and treatment of inflammatory-associated diseases.

\section{Acknowledgment}

This work was supported by grants from the National Research Foundation of Korea (NRF-2017R1A2B4009301 and MRC-2009-0083533).

\section{References}

(1) Fujiwara, N.; Kobayashi, K. Curr. Drug Targets Inflamm. Allergy 2005, 4, 281-286.

(2) Amin, A. R.; Attur, M.; Abramson, S. B. Curr. Opin. Rheumatol. 1999, 11, 202-209.

(3) Tak, P. P.; Firestein, G. S. J. Clin. Invest. 2001, 107, 7-11.

(4) Yamamoto, Y.; Gaynor, R. B. Trends Biochem. Sci. 2004, 29, 72-79.

(5) Yuan, G.; Wahlqvist, M. L.; He, G.; Yang, M.; Li, D. Asia Pac. J. Clin. Nutr. 2006, 15, 143-152.

(6) Spiridon, I.; Nechita, C. B.; Niculaua, M.; Silion, M.; Armatu, A.; Teaca, C. A.; Bodirlau, R. Cent. Eur. J. Chem. 2013, 11, 1699-1709.
(7) Trendafilova, A.; Chanev, C.; Todorova, M. Pharmacogn. Mag. 2010, 6, 234-237.

(8) Babaei, G.; Aliarab, A.; Abroon, S.; Rasmi, Y.; Aziz, S. G. Biomed. Pharmacother. 2018, 106, 239-246.

(9) Chun, J.; Choi, R. J.; Khan, S.; Lee, D. S.; Kim, Y. C.; Nam, Y. J.; Lee, D. U.; Kim, Y. S. Int. Immunopharmacol. 2012, 14, 375-383.

(10) He, G.; Zhang, X.; Chen, Y.; Chen, J.; Li, L.; Xie, Y. Biomed. Pharmacother. 2017, 90, 598-607.

(11) Ketai, W.; Huitao, L.; Yunkun, Z.; Xingguo, C.; Zhide, H.; Yucheng, S.; Xiao, M. Talanta 2000, 52, 1001-1005.

(12) Gao, S.; Wang, Q.; Tian, X. H.; Li, H. L.; Shen, Y. H.; Xu, X. K.; Wu, G. Z.; Hu, Z. L.; Zhang, W. D. J. Ethnopharmacol. 2017, 196, 39-46.

(13) Engström, M. T.; Pälijärvi, M.; Salminen, J. P. J. Agric. Food Chem. 2015, 63, 4068-4079.

(14) Ahn, K. S.; Noh, E. J.; Zhao, H. L.; Jung, S. H.; Kang, S. S.; Kim, Y. S. Life Sci. 2005, 76, 2315-2328.

(15) Kim, S. F.; Huri, D. A.; Snyder, S. H. Science 2005, 310, 19661970.

(16) Tang, X.; Liu, D.; Shishodia, S.; Ozburn, N.; Behrens, C.; Lee, J. J.; Hong, W. K.; Aggarwal, B. B.; Wistuba, I. I. Cancer 2006, 107, 26372646.

(17) Morris, C. J. Methods Mol. Biol. 2003, 225, 115-121.

(18) Guo, C.; Zhang, S.; Teng, S.; Niu, K. J. Sep. Sci. 2014, 37, 950956.

(19) Calixto, J. B. Braz. J. Med. Biol. Res. 2000, 33, 179-189.

Received July 16, 2018 Revised September 6, 2018 Accepted September 7, 2018 